\title{
Nonequivalent lanthanide defects: energy level modeling
}

\author{
Jonas J. Joos, Dirk Poelman, Philippe F. Smet
}

LumiLab, Department of Solid State Sciences, Ghent University, Krijgslaan 281-S1, 9000 Gent, Belgium.

Center for Nano- and Biophotonics (NB Photonics), Ghent University, Belgium

This paper was published in Opt. Mater. (Available online 15 May 2016)

This version is the unedited, accepted version after peer review.

The edited version can be found at:

http://www.sciencedirect.com/science/article/pii/S0925346716301446

Optical Materials 61 (2016) 50-58

Contents lists available at ScienceDirect

Optical Materials

journal homepage: www.elsevier.com/locate/optmat

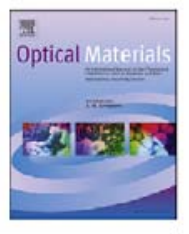

Nonequivalent lanthanide defects: Energy level modeling

Jonas J. Joos ${ }^{\mathrm{a}, \mathrm{b}}$, Dirk Poelman a, b, Philippe F. Smet a, b, *

CrossMark 


\title{
Nonequivalent lanthanide defects: energy level modeling
}

\author{
Jonas J. Joos, Dirk Poelman, Philippe F. Smet ${ }^{1}$ \\ LumiLab, Department of Solid State Sciences, Ghent University, Belgium \\ Center for Nano- and Biophotonics (NB Photonics), Ghent Univeristy, Belgium
}

\begin{abstract}
Empirical charge-state transition level schemes are popular tools to model the properties of lanthanide-doped materials and their construction has become standard practice. Typically, it is implicitly assumed that all lanthanide ions form isostructural defects. However, in practice, multiple nonequivalent defects related to the same lanthanide can occur or different lanthanides can even incorporate in different ways. The consequences of these complications on the impurity energy levels are discussed in this article. It seems that small structural differences around the lanthanide dopant can give rise to important spectral differences in its emission. These are not always clearly reproduced by the charge-state transition level schemes. Improvements to the existing procedure are suggested and applied to the lanthanide ions in the well-studied host crystals $\mathrm{SrAl}_{2} \mathrm{O}_{4}, \mathrm{Sr}_{2} \mathrm{Si}_{5} \mathrm{~N}_{8}$ and $\mathrm{SrGa}_{2} \mathrm{~S}_{4}$.
\end{abstract}

\section{Introduction}

Transparent inorganic solids have a long history of optical activation by crystallographic defects. Gems that are colored by metal impurities or color centers are well-known in mineralogy while optical materials based on intentionally doped dielectrics are integral parts of modern technologies such as lighting, electronic displays, medical imaging etc [1, 2, 3, 4, 5, 6. In these cases, the defects are mostly structurally simple point defects such as a missing atom, i.e. a vacancy, or an impurity atom in place of a normal atom, i.e. a substitutional defect [7]. In the following, defects which are induced by doping a crystal with lanthanide ions are considered.

Since the emergence of quantum mechanical theories, spectroscopy has been associated with energy level schemes, representing a connection between the experimental observables, i.e. the spectra, and the fundamental interactions. For this reason, understanding the physical origin of the luminescence behavior of a particular material often boils down to constructing an energy levels scheme. The distinct energy levels are suitably labeled, encoding for the dominant interactions. Furthermore, selection rules and transition probabilities are governed by the interaction of the physical system with the ambient electromagnetic field, i.e. incident photons.

Two important classes of energy level schemes exist [8]. Firstly, there are many-body schemes in which the energy level represents the total energy of the complete system. This means that not only the electrons which are considered as spectroscopically active are included, but also the other electrons of the system. Formally, spectroscopic transitions can only be correctly described in a many-body framework. This is especially true in the case of transition metal or lanthanide ions due to the important electron correlations in these systems, i.e. all electrons of the system are to a certain extent affected by the excitation 9. Secondly, in single particle energy level schemes, individual electrons are assigned to individual (spin)orbitals. In some systems, a single (quasi)particle picture is able to describe the electronic structure, yielding single particle states that are either occupied or empty. An example are the valence and conduction bands of a semiconductor 10.

In order to avoid the need of the explicit calculation of the electronic structure and optical properties of these materials, trends have been described, yielding not only predictability, but also empirical energy level schemes [11. The nature of the empirical energy level schemes under discussion requires some attention as they should be envisioned as a separate tool with respect to the above-mentioned single-particle or many-body energy level schemes. Essentially, a singleparticle picture of the host material, i.e. the valence and conduction bands, are utilized and impurity levels of the lanthanide are defined as so-called charge-state transition levels. These correspond to the value of the electronic chemical potential at which the formal charge of the lanthanide ion changes [12. The exact meaning of the empirical energy levels is further elucidated in [8].

Lanthanide ions show a particular electronic structure. The incompletely filled $4 \mathrm{f}$ shell is effectively screened by the environment by filled 5 s and 5 p shells. Furthermore, some lanthanide ions show a low-lying $4 \mathrm{f}^{N-1} 5 \mathrm{~d}^{1}$ excited configuration, allowing efficient excitation with visible or near ultraviolet light. These particular features give rise to a systematic behavior of the ionization potentials and 4f-5d excitation energies across the lanthanide series which can be exploited to devise empirical rules with predictive 
power. This was pioneered by Thiel in the context of photoelectron spectroscopy and further developed by Dorenbos, mostly based on optical spectroscopy [13, 14, 15, 11. The uncertainties associated with these empirical models and the accuracy by which experimental quantities can be predicted have recently been addressed in detail 8 .

Up to now, the described empirical rules and techniques for constructing charge-state transition level schemes have been applied to many host materials [16, 17, 18, 19, 20, 21, 22, 23. This can be unambiguously done for host materials in which all lanthanides act identically in chemical and crystallographic terms. One can expect that often this is true because of their similar ionic radii and chemical behavior. However examples are known where this is not the case. Furthermore, there is a one-to-one correspondence between the number of nonequivalent defects in a certain host and the number of charge-state transition levels for a dopant. If multiple defect geometries for the same foreign atom are possible, multiple energy level schemes are necessary for a correct description. Both these subtleties are often neglected or simply forgotten.

In this work, the empirical energy level models are applied to lanthanide-doped materials with more challenging host compounds, featuring multiple nonequivalent lattice sites on which the lanthanides can incorporate. $\mathrm{SrAl}_{2} \mathrm{O}_{4}$ and $\mathrm{Sr}_{2} \mathrm{Si}_{5} \mathrm{~N}_{8}$ are presented as case studies. Site-selective spectroscopy can offer a tidy opportunity to separate the spectral features and construct charge-state transition schemes for the different defect geometries. Remaining difficulties such as correlating the spectral features to the defects are discussed.

In the second part of this article, recent work on the lanthanide doped ternary sulfide $\mathrm{SrGa}_{2} \mathrm{~S}_{4}$ is presented. It is known that the first coordination shell is severely altered when $\mathrm{Ce}^{3+}$ is incorporated 24, yielding a completely different situation than for $\mathrm{Eu}^{2+}$ doping. In this case, the use of empirical rules that relate spectral properties of different lanthanides becomes severely restricted. The repercussions on the construction of charge-state transition level schemes are discussed in detail.

\section{Experimental}

In this manuscript, lanthanide defects are described in three host materials, i.e. $\mathrm{SrAl}_{2} \mathrm{O}_{4}, \mathrm{Sr}_{2} \mathrm{Si}_{5} \mathrm{~N}_{8}$ and $\mathrm{SrGa}_{2} \mathrm{~S}_{4}$. The discussion of the former two is based on literature data, the discussion of the latter is based on following experiments.

$\mathrm{SrGa}_{2} \mathrm{~S}_{4}: \operatorname{Ln}^{Q+}$ powders were synthesized by a solid state reaction at high temperature $\left(2\right.$ hours at $900^{\circ} \mathrm{C}$, heating rate of $7.5^{\circ} \mathrm{C} / \mathrm{min}$ ) under a flow of forming gas $\left(90 \% \mathrm{~N}_{2}, 10 \% \mathrm{H}_{2}\right)$ with $\mathrm{SrS}$ (Alfa Aesar, 99.9\%) and $\mathrm{Ga}_{2} \mathrm{~S}_{3}$ (Alfa Aesar, 99.99\%) as starting materials for the host crystal and lanthanide fluorides $\left(\mathrm{LnF}_{3}\right.$, all at least $99.5 \%$ pure) as source of the dopants. The selected dopants are Ce, Pr, Sm, Eu, Tb and Tm. A doping concentration of $1 \mathrm{~mol} \%$ was used for all syntheses.

The phase purity of the obtained powders was verified by means of X-ray diffraction (XRD) with $\mathrm{CuK} \alpha_{1}$ radiation on a Bruker D5000 diffractometer $(40 \mathrm{kV}, 40 \mathrm{~mA})$.

Luminescence emission and excitation spectra were measured with an Edinburgh FS920 fluorescence spectrometer. Spectra at low temperature were obtained by using an Oxford Optistat CF cryostat.

Diffuse reflectance spectra were measured with a Varian Cary 500 spectrophotometer, equipped with an internal integrating sphere, using $\mathrm{BaSO}_{4}$ as a reference. From the reflectance spectra, the square of the Kubelka-Munk function was calculated and fitted with a straight line to obtain an estimate for the optical band gap.

\section{Results and discussion}

\subsection{Multiple nonequivalent lanthanide defects}

In this paragraph, two examples of host compounds $\left(\mathrm{SrAl}_{2} \mathrm{O}_{4}\right.$ and $\left.\mathrm{Sr}_{2} \mathrm{Si}_{5} \mathrm{~N}_{8}\right)$ are discussed which feature two nonequivalent substitutional defects upon lanthanide doping. This poses additional difficulties when the electronic structure is empirically assessed as both lanthanide defects require a separate energy level scheme. Examples are included for which the occurrence of the nonequivalent defects is clear from the optical spectra, i.e. site-selective spectroscopy can be performed. This is preferably done on low concentrated systems, avoiding interactions between nonequivalent defect centers. Interactions between centers result in energy transfer which can obscure the optical spectra.

Site-selective spectroscopy of the $\mathrm{Ce}^{3+}$ ion is an important first step as it allows to construct vacuum referred binding energy level schemes for the $4 \mathrm{f}$ levels. This is thanks to an empirical rule due to Dorenbos, relating the centroid shift of the $\mathrm{Ce}^{3+} 5 \mathrm{~d}^{1}$ manifold to the vacuum referred binding energies (VRBE) of all lanthanides [25, 26]. This procedure, in which the empirical Coulomb correlation energy is used as intermediate parameter is described in two review papers [11, 8]. In this way, the $4 \mathrm{f}$ ionization potentials of the different lanthanide defects are obtained with a reasonable accuracy [8].

Next, the host compound's valence and conduction 
band need to be added to obtain a complete chargestate transition level diagram and the host referred binding energies (HRBE). From photoluminescence excitation spectroscopy of a trivalent lanthanide, the anion to lanthanide charge-transfer (CT) energy can be obtained, approximately probing the $2+/ 3+$ charge-state transition level. The binding energy difference between two nonequivalent $\mathrm{Ln}^{3+}$ defects is significantly smaller than the typical width of a charge-transfer spectral band [27]. As the ionization potentials for both defects were already calculated, deconvolution of the CT band poses no additional problems.

\subsection{1 $\mathrm{SrAl}_{2} \mathrm{O}_{4}$}

Doped with divalent europium, strontium aluminate $\left(\mathrm{SrAl}_{2} \mathrm{O}_{4}\right)$ features an extensively studied green afterglow which can be intensified upon codoping with trivalent dysprosium [28, 29, 30]. Interestingly, when this material is cooled below $250 \mathrm{~K}$, a second emission band emerges in the blue spectral region [31. The origin of this band has been the debated since it was first found. Although an explanation in terms of incorporation of $\mathrm{Eu}^{2+}$ on the two nonequivalent lattice sites seems the most simple one, it was plead that the minor geometrical differences between the Sr1 and Sr2 sites cannot account for the differences between the two spectral features [32, 33, 34, 35]. Alternatively, more exotic explanations such as alignment of Eu ions, hole release or even emission from a higher $4 \mathrm{f}^{6} 5 \mathrm{~d}^{1}$ multiplet were contrived [32, 34, 35].

Recently, empirical rules, relating the bond lengths of the undistorted lattice sites to the multiplet structure of the excited $4 \mathrm{f}^{6} 5 \mathrm{~d}^{1}$ configuration were applied, showing that the order of magnitude of the spectral differences between the blue and green emission bands can be perfectly explained by the structural differences between both lattice sites, discarding the more exotic explanations for the occurrence of two emission bands [31.

Also upon $\mathrm{Ce}^{3+}$ doping, it was spectroscopically shown that two different defects are present 36]. Based on this data, which is summarized in Tab. 1. chargestate transition level schemes can be constructed. The $2+/ 3+$ levels for all the lanthanides, i.e. the divalent 4f curves, are shown in Fig. 1 .

\subsection{2 $\quad \mathrm{Sr}_{2} \mathrm{Si}_{5} \mathrm{~N}_{8}$}

Strontium nitridosilicate, $\mathrm{Sr}_{2} \mathrm{Si}_{5} \mathrm{~N}_{8}$, has been frequently reported as a chemically stable host material for lanthanides. In particular, upon doping with divalent europium, an efficient red phosphor is formed which is applied in phosphor-converted white lightemitting diodes (LEDs) 39, 40, 41, 42, 43, 44, 45.

Spectroscopy of $\mathrm{Ce}^{3+}$ in $\mathrm{Sr}_{2} \mathrm{Si}_{5} \mathrm{~N}_{8}$ was performed by $\mathrm{Li}$ et al. They clearly found the effect of two

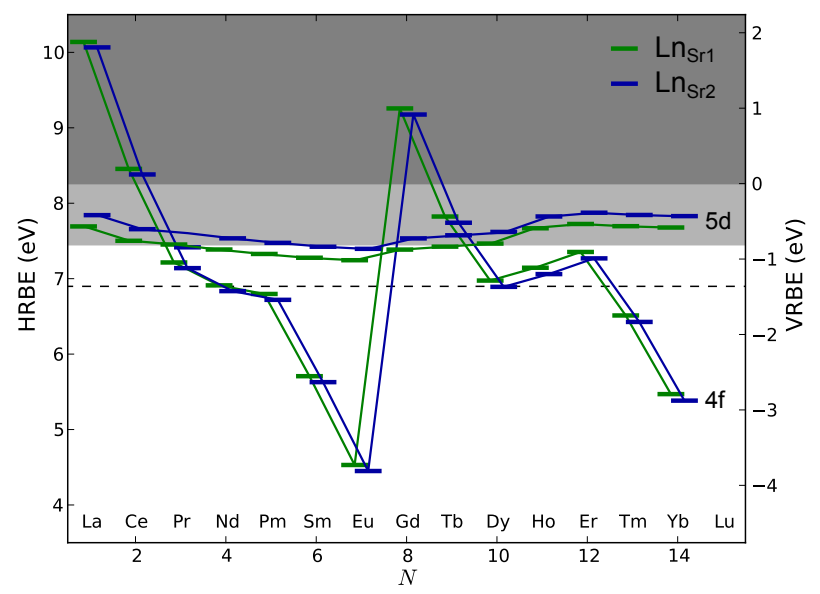

Figure 1 - Diagram with the $2+/ 3+$ charge-state transition levels for the $\operatorname{Ln}_{\mathrm{Sr} 1}$ and $\mathrm{Ln}_{\mathrm{Sr} 2}$ defects in $\mathrm{SrAl}_{2} \mathrm{O}_{4}$. Adapted from 31, reproduced with permission.

nonequivalent cerium defects in their spectra of which different crystal field splitting energies could be extracted [37]. Based on their data, the centroid shifts of the cerium $5 \mathrm{~d}^{1}$ configuration for both cerium defects were estimated. The data is summarized in table 1 .

More recently, Lazarowska et al. performed a detailed site-selective spectroscopy study of the $\mathrm{Eu}^{2+}$ ion in $\mathrm{Sr}_{2} \mathrm{Si}_{5} \mathrm{~N}_{8}$ 38. From the $4 \mathrm{f}^{7}-4 \mathrm{f}^{6} 5 \mathrm{~d}^{1}$ excitation spectra, it was concluded that the spectroscopic redshift for both europium defects are very similar. The difference in emission energy is therefore almost completely due to a different electron-vibrational interaction. Empirical total energy level schemes within the single configurational coordinate approximation were provided.

Based on site-selective spectroscopy on $\mathrm{Ce}^{3+}$ and $\mathrm{Eu}^{2+}$ and the charge transfer energy of $\mathrm{Sm}^{3+}$ [46], charge-state transition level schemes were constructed for both defect geometries (see Fig. 2).

An empirical charge-state transition level scheme for lanthanide defects in $\mathrm{Sr}_{2} \mathrm{Si}_{5} \mathrm{~N}_{8}$ was first constructed in 2013 by ten Kate et al. 46. Only the charge-state transition levels for the low energy site were calculated. Though not represented in the energy level scheme, the $4 \mathrm{f}^{N}-4 \mathrm{f}^{N-1} 5 \mathrm{~d}^{1}$ transition energies for lanthanides were compared for both defect configurations. Interestingly, for the $\mathrm{Tb}^{3+}$ ion only one $4 \mathrm{f}^{8}-4 \mathrm{f}^{7} 5 \mathrm{~d}^{1}$ excitation band was found. By extrapolating the $4 \mathrm{f}-5 \mathrm{~d}$ energies of the cerium defects according to the empirical finding that all $\mathrm{Ln}^{3+}$ ions feature the same $4 \mathrm{f}-5 \mathrm{~d}$ spectroscopic redshift, the excitation band was attributed to the Sr1 site. This means that the $\mathrm{Tb}^{3+}$ ion occupies preferentially the smaller Sr1 site while no preference was found for the slightly larger $\mathrm{Ce}^{3+}$ ion [46].

Inspection of the diagram in Fig. 2 learns that the binding energies for both defect sites are very close. 
Table 1 - Experimental spectroscopic parameters for lanthanides incorporated on the nonequivalent Sr sites in $\mathrm{SrAl}_{2} \mathrm{O}_{4}$ and $\mathrm{Sr}_{2} \mathrm{Si}_{5} \mathrm{~N}_{8} . \epsilon_{\mathrm{cfs}}, \epsilon_{\mathrm{c}}$ and $D$ denote respectively the crystal field splitting, centroid shift and redshift of the $4 \mathrm{f}^{N-1} 5 \mathrm{~d}^{1}$ manifolds. $\Delta S$ denotes the Stokes shift of the $4 \mathrm{f}^{N}-4 \mathrm{f}^{N-1} 5 \mathrm{~d}^{1}$ luminescence. All values in $\mathrm{eV}$.

\begin{tabular}{|c|c|c|c|c|c|c|}
\hline & & $\begin{array}{l}\mathrm{SrAl}_{2} \mathrm{O}_{4} \\
\text { low energy }\end{array}$ & high energy & $\begin{array}{l}\mathrm{Sr}_{2} \mathrm{Si}_{5} \mathrm{~N}_{8} \\
\text { low energy }\end{array}$ & high energy & ref. \\
\hline \multirow[t]{4}{*}{$\mathrm{Ce}^{3+}$} & $\epsilon_{\mathrm{cfs}}$ & 0.85 & 1.14 & 1.91 & 1.61 & \begin{tabular}{l|l|l}
36 & 31 & 37 \\
\end{tabular} \\
\hline & $\epsilon_{\mathrm{C}}$ & 2.65 & 2.23 & 2.73 & 2.41 & 36, 31, 37. \\
\hline & $D$ & 2.85 & 2.47 & 3.24 & 3.02 & 36, 31, 37 \\
\hline & $\Delta S$ & 0.25 & 0.31 & 0.33 & 0.87 & 36, 31,37 \\
\hline \multirow[t]{2}{*}{$\mathrm{Eu}^{2+}$} & $D$ & 1.42 & 1.22 & 2.05 & 2.02 & 31,38 \\
\hline & $\Delta S$ & 0.43 & 0.21 & 0.32 & 0.23 & 31, 38 \\
\hline
\end{tabular}

How can this be compatible with the undeniable spectral differences between both types of defects? This is a direct consequence of the fact that these empirical charge-state transition level schemes do not take vibronic interactions or correlation (i.e. multiplet) effects into account [47, 8. In the case of $\mathrm{Ce}^{3+}$, there is a clear difference in the details of the $5 \mathrm{~d}$ manifold for both defects. This is dictated by the exact details of the crystal field interaction, which is not described by the charge-state transition levels. In the case of $\mathrm{Eu}^{2+}$, the spectral difference is a direct consequence of a different local electron-vibration interaction. To correctly describe the dynamics, details of the potential energy surfaces, such as curvatures, equilibrium coordinates and relevant normal modes are indispensable. No empirical rules to obtain these quantities are available to date.

In [38, it was argued that the preferential incorporation of $\mathrm{Eu}$ on the site yielding the higher-energy emission is due to the $4 \mathrm{f}$ level of this defect being more strongly bound than for the alternative defect. This is however an incorrect interpretation of the chargestate transition levels. It is true, by definition of the charge-state transfer energies, that the $\mathrm{Eu}_{\mathrm{Sr} 2}$ defect requires slightly more energy in order to oxidize. On the other hand, preferential occupation of certain lattice sites is governed by the defect formation energy which is calculated in terms of total energies instead of singleparticle energies [12]. Furthermore, if the difference in defect formation energies would be the same as the difference in charge-state transition levels, it would be too small to yield an observable difference in site occupation given the high temperature at which the materials are synthesized.

\subsubsection{Relating crystal sites to spectra}

The remaining challenge is linking the separate spectral features to the different defect geometries. It is often assumed that a smaller lattice site corresponds to a lower $4 \mathrm{f}-5 \mathrm{~d}$ energy. The reasoning is that the crystal field strength, and implicitly the spectroscopic redshift of the $5 \mathrm{~d}$ level, increases upon decreasing bond lengths. The validity of this assumption is doubtful for lattice sites for which the geometry does not differ too much. Moreover, the crystal lattice will locally relax to a different equilibrium geometry, adapted to the specific lanthanide atom in an unknown way.

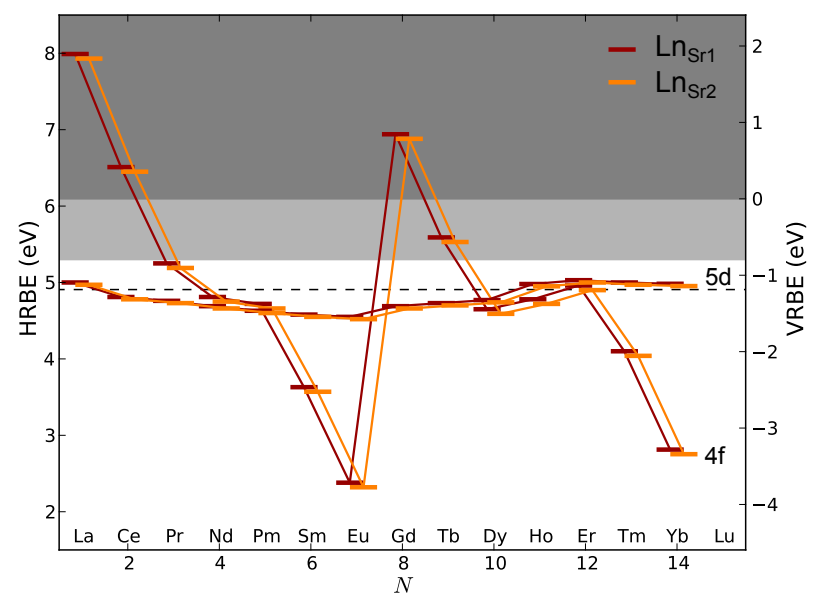

Figure 2 - Diagram with the $2+/ 3+$ charge-state transition levels for the $\operatorname{Ln}_{\mathrm{Sr} 1}$ and $\mathrm{Ln}_{\mathrm{Sr} 2}$ defects in $\mathrm{Sr}_{2} \mathrm{Si}_{5} \mathrm{~N}_{8}$. Assignation according to 37] and 38.

As the different lanthanide ions behave very similar in chemical terms, it is likely that high- and low-energy sites can be correlated for the different lanthanides, allowing to construct empirical charge-state transition diagrams for both defects. Exceptions to this rule of thumb are discussed in $\$ 3.2$.

$\mathrm{SrAl}_{2} \mathrm{O}_{4}$ forms monoclinic crystals (space group $\mathrm{P} 2_{1}$ ) in which two nonequivalent $\mathrm{Sr}$ sites occur, both on Wyckoff position 2a. $\mathrm{Sr}_{2} \mathrm{Si}_{5} \mathrm{~N}_{8}$ forms orthorhombic crystals (space group $\mathrm{Pmn}_{1}$ ) [4] in which likewise two nonequivalent $\mathrm{Sr}$ sites occur, both corresponding with Wyckoff site 2a [48].

In $\mathrm{Sr}_{2} \mathrm{Si}_{5} \mathrm{~N}_{8}$, the $\mathrm{Sr} 1$ site has a lower coordination number and features a smaller polyhedron than the $\mathrm{Sr} 2$ site 48. Specifying the coordination number, i.e. the number of next neighbor atoms is somewhat arbitrary in these cases. This is illustrated in literature where different coordination numbers are reported for the same compound 37, 46, 38.

The reason for the ambiguity is demonstrated in Fig. 4 where the number of anions lying inside a sphere, centered at the $\mathrm{Sr}$ atom is given as a function of the radius of the sphere. This figure shows that coordination shells cannot be identified in a straightforward fashion, neither for $\mathrm{SrAl}_{2} \mathrm{O}_{4}$ nor $\mathrm{Sr}_{2} \mathrm{Si}_{5} \mathrm{~N}_{8}$. In the case of $\mathrm{SrGa}_{2} \mathrm{~S}_{4}$, a higher symmetry is present and 


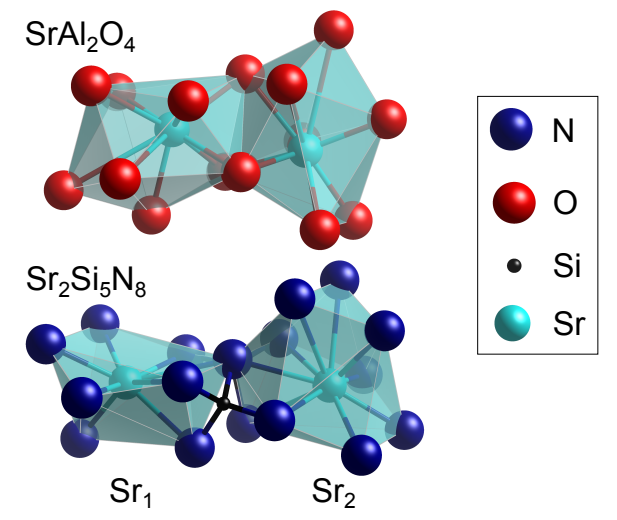

Figure 3 - Coordination environments for the $\mathrm{Sr} 1$ and $\mathrm{Sr} 2$ sites in $\mathrm{Sr}_{2} \mathrm{Si}_{5} \mathrm{~N}_{8}$ (top) and $\mathrm{SrAl}_{2} \mathrm{O}_{4}$ (bottom). Crystal data from 48, 49.

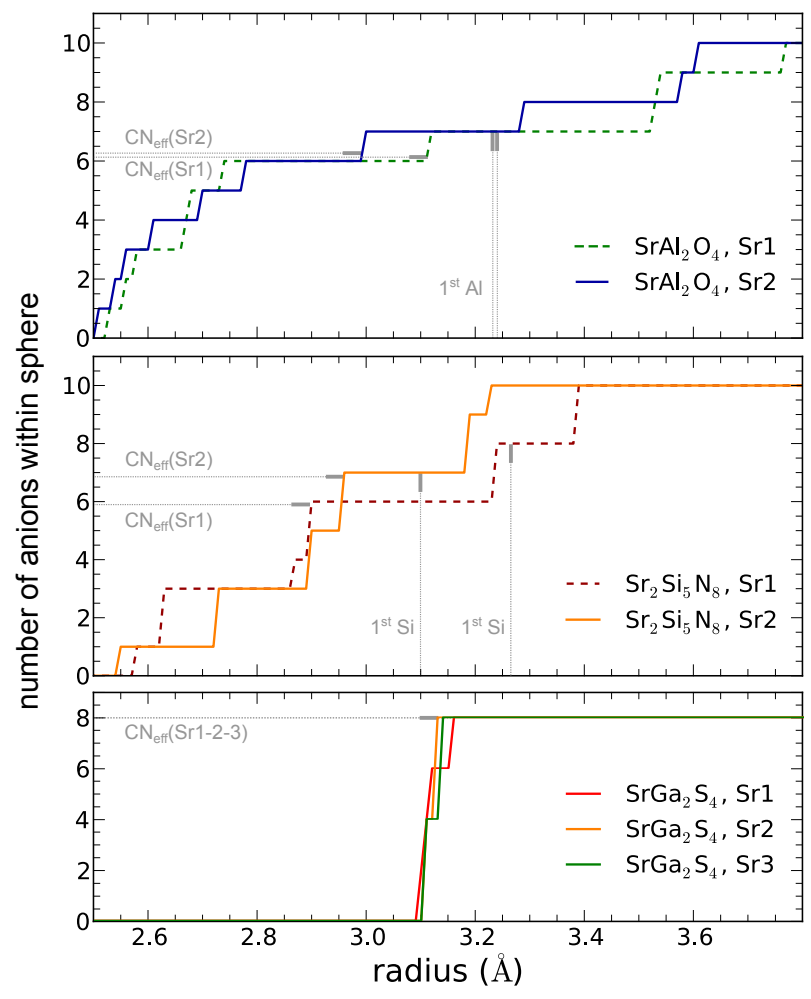

Figure 4 - Illustration of the number of anions (O, N or S) contained inside a sphere centered at the cationic $(\mathrm{Sr})$ sites as a function of the radius of the sphere for $\mathrm{SrAl}_{2} \mathrm{O}_{4}$ (top), $\mathrm{Sr}_{2} \mathrm{Si}_{5} \mathrm{~N}_{8}$ (middle) and $\mathrm{SrGa}_{2} \mathrm{~S}_{4}$ (bottom). The effective coordination number, as defined by R. Hoppe, is given for each site 50. Furthermore, the location of the closest cation $(\mathrm{Al}, \mathrm{Si})$ is given. The smallest Sr-Ga distance in $\mathrm{SrGa}_{2} \mathrm{~S}_{4}$ is $3.86 \stackrel{\circ}{A}$. well-defined coordination shells can be identified.

To straighten out this ambiguity, Rudolf Hoppe introduced the effective coordination number in 1979 in which a weighing of the different bond lengths is carried out [50]. This quantity turns out to be simply the ordinary coordination number in the case of compounds with a high symmetry and well-defined coordination shells, while it yields a rational number in other cases. The effective coordination numbers for the $\mathrm{Sr} 1$ and $\mathrm{Sr} 2$ sites of $\mathrm{Sr}_{2} \mathrm{Si}_{5} \mathrm{~N}_{8}$ are respectively 5.98 and 6.88. The coordination polyhedra of the first 6 and 7 neighbors for the $\mathrm{Sr} 1$ and $\mathrm{Sr} 2$ sites of $\mathrm{Sr}_{2} \mathrm{Si}_{5} \mathrm{~N}_{8}$ are approximate pentagonal and hexagonal pyramids respectively [48].

Several assignments of the spectral bands of $\mathrm{SrAl}_{2} \mathrm{O}_{4}: \mathrm{Ln}^{Q+}$ are available in literature. Jia et al. showed that the high energy band in $\mathrm{SrAl}_{2} \mathrm{O}_{4}: \mathrm{Ce}^{3+}$ has an emission intensity which is 10 times higher than the intensity for the low energy band at room temperature [36]. From this result, it was concluded that the high energy emission originates from Ce incorporated on the larger Sr1 site as this provides more space for the suggested charge compensation [36. This is however a somewhat arbitrary argument because the intensity of an emission band is not necessarily related to a preferential site occupation and can as well be the result of a different thermal quenching behavior.

Botterman et al. used empirical rules, relating bond lengths in the defect cluster to spectral characteristics of $\mathrm{Eu}^{2+}$ to assign the spectral bands to the $\mathrm{Sr}$ sites 31. These rules showed that the high energy emission is most likely attributed to the smaller $\mathrm{Sr} 2$ site.

More recently, vacuum UV spectroscopy of $\mathrm{Sr}_{0.75} \mathrm{Ce}_{0.125} \mathrm{Na}_{0.125} \mathrm{Al}_{2} \mathrm{O}_{4}$ was combined with quantum mechanical calculations to gain a better understanding of the connection between defect geometry and spectral properties [51]. The sodium atoms were added for charge-compensating the $\mathrm{Ce}_{\mathrm{Sr}}^{\bullet}$ defects and located on the nearest or next-nearest $\mathrm{Sr}$ site for the calculations. The multireference calculations confirmed that the two spectral features originate from incorporation on the different $\mathrm{Sr}$ sites. The allocation of the $\mathrm{Ce}_{\mathrm{Sr} 1}^{\bullet}$ and $\mathrm{Ce}_{\mathrm{Sr} 2}^{\bullet}$ defects to respectively the low and high energy emission bands was however reversed by the calculation. It still remains a question why the $4 \mathrm{f}-5 \mathrm{~d}$ transition of $\mathrm{Ce}^{3+}$ on the larger $\mathrm{Sr}$ site would correspond to the highest energy. Additionally, the influence of the nearby $\mathrm{Na}_{\mathrm{Sr}}^{\prime}$ defect on the multiplets of the $\mathrm{Ce}^{3+}$ ion is unclear as the Coulomb interactions between the different defects can have a rather long range 7].

In the case of $\mathrm{Sr}_{2} \mathrm{Si}_{5} \mathrm{~N}_{8}: \mathrm{Eu}^{2+}$, the lowest energy emission was attributed to the smallest undistorted $\mathrm{Sr}$ site according to the typical rule of thumb 37, 38. It was found that the $\mathrm{Eu}^{2+}$ ion occupies both $\mathrm{Sr}$ sites, although it has a preference for the larger $\mathrm{Sr} 2$ site 
because it is the only occupied site for low doping concentrations. In the article of Lazarowka et al., the labeling of the Sr1 and Sr2 sites was exchanged with respect to the assignation by Schlieper et al. [48 which is used in this article, following the convention of Yeh et al. [52.

From these examples, it is clear that the exact way by which subtle differences in defect geometry can result in appreciable spectral changes are still very difficult to predict.

\subsection{Structural distortions across the lanthanide series; the example of $\mathrm{SrGa}_{2} \mathrm{~S}_{4}$}

Previous examples, $\mathrm{SrAl}_{2} \mathrm{O}_{4}$ and $\mathrm{Sr}_{2} \mathrm{Si}_{5} \mathrm{~N}_{8}$, were challenging host compounds for constructing empirical charge-state transition level schemes in the sense that two nonequivalent lanthanide defects occurred and formally, two different schemes are required. Yet, because site selective spectroscopy allows to separate the different spectral features, the two schemes can be constructed almost independently.

A more complex situation occurs if not all lanthanides behave in the same way with respect to incorporation. If this is the case, the systematics which are normally exploited in the construction of the chargestate transition level schemes break down and it should be carefully considered which empirical rules are still usable. $\mathrm{SrGa}_{2} \mathrm{~S}_{4}$ is an example of such a host compound.

\subsubsection{Crystal structure}

Strontium thiogallate $\left(\mathrm{SrGa}_{2} \mathrm{~S}_{4}\right)$ forms crystals with orthorhombic unit cells (space group Fddd). The strontium atoms are situated on three nonequivalent Wyckoff sites, 8a, 8b and 16e. The former two feature $D_{2}$ site symmetry, the latter $C_{2}$ site symmetry (see Fig. 5). However, all three $\mathrm{Sr}$ sites do only differ very slightly which is demonstrated in Fig. 4. Therefore, this compound is considered to show only one type of $\mathrm{Sr}$ site in the unit cell. Furthermore, the geometry is very close to square antiprismatic. For this reason, a higher $D_{4 \mathrm{~d}}$ effective symmetry is often utilized. $\mathrm{Eu}^{2+}$ doping is expected to deform the coordination polyhedron only minimally due to the comparable ionic radii of $\mathrm{Eu}^{2+}$ and $\mathrm{Sr}^{2+}$ and given that $\mathrm{EuGa}_{2} \mathrm{~S}_{4}$ is isostructural with $\mathrm{SrGa}_{2} \mathrm{~S}_{4}$ [53, 54, 55. Doped with $\mathrm{Eu}^{2+}$ or $\mathrm{Ce}^{3+}$, this compound has been thoroughly described in view of multiple applications such as electroluminescent displays, field-emissive devices and more recently LED phosphor [56, 57, 58, 59, 60, 61.

Although this compound effectively features only one $\mathrm{Sr}$ site and might therefore be considered as a straightforward case upon lanthanide doping, a discontinuity across the lanthanide series occurs.

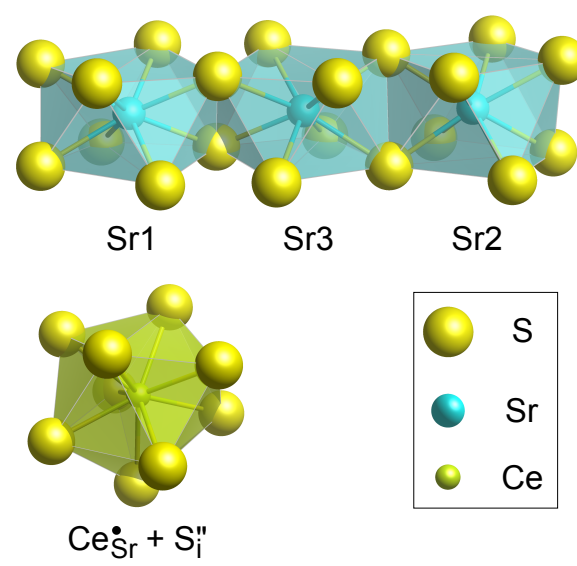

Figure 5 - Coordination environments for the $\mathrm{Sr}$ sites in $\mathrm{SrGa}_{2} \mathrm{~S}_{4}$ (top) and for the $\mathrm{Ce}_{\mathrm{Sr}}^{\bullet}$ defect in $\mathrm{SrGa}_{2} \mathrm{~S}_{4}$ (bottom) 62, 24].

Warren et al. have shown by electron paramagnetic resonance (EPR) spectroscopy that the $\mathrm{Ce}_{\mathrm{Sr}}^{\bullet}$ defect induces a sulfur interstitial $\mathrm{S}_{\mathrm{i}}^{\prime \prime}$ in the first coordination shell to compensate the excess positive charge [24]. The deformed coordination polyhedron for the $\mathrm{Ce}^{3+}$ dopant features approximate $C_{3 \mathrm{~h}}$ point symmetry and a coordination number of 9 (see Fig. 5).

The geometrical deviation for the $\mathrm{Ce}_{\mathrm{Sr}}^{\bullet}$ defect is expected to have an influence on the luminescence properties. It is self-evident that this has to be taken into account when constructing charge-state transition level schemes. In the following, the optical spectra and electronic structure of $\mathrm{SrGa}_{2} \mathrm{~S}_{4}: \mathrm{Ln}^{Q+}$ will be compared to $\mathrm{CaGa}_{2} \mathrm{~S}_{4}: \mathrm{Ln}^{Q+}$. Both compounds are isostructural and it has been shown by EPR that for $\mathrm{CaGa}_{2} \mathrm{~S}_{4}: \mathrm{Ln}^{Q+}$ the pristine symmetry and coordination number of the Sr site is maintained upon $\mathrm{Ce}^{3+}$ doping which might be due to the similar ionic radii of $\mathrm{Ce}^{3+}$ and $\mathrm{Ca}^{2+} 63$. For this reason, $\mathrm{CaGa}_{2} \mathrm{~S}_{4}: \mathrm{Ln}^{Q+}$ is a good choice as a well-behaving reference.

\subsubsection{Photoluminescence}

Fig. 6(a-b-c) shows the emission and excitation spectra of $\mathrm{Eu}^{2+}, \mathrm{Ce}^{3+}$ and $\mathrm{Tm}^{3+}$ in $\mathrm{SrGa}_{2} \mathrm{~S}_{4}$, compared to the spectra of the same lanthanides in $\mathrm{CaGa}_{2} \mathrm{~S}_{4}$. The spectra of these three defects can be used as input to construct the empirical charge-state transition level schemes. This was done for $\mathrm{CaGa}_{2} \mathrm{~S}_{4}$ in 8 . The numerical values of the parameters that can be obtained from these measurements are summarized in Table 1.

For both host compounds, $\mathrm{Eu}^{2+}$ and $\mathrm{Ce}^{3+}$ show the typical broadband luminescence, originating from interconfigurational $4 \mathrm{f}^{N}-4 \mathrm{f}^{N-1} 5 \mathrm{~d}$ transitions. $\mathrm{Tm}^{3+}$ features near-infrared line emission, originating from intraconfigurational $4 \mathrm{f}^{12}-4 \mathrm{f}^{12}$ transitions. The emission can be efficiently excited by a charge transfer transition, yielding a broad excitation band in the UV region. In the $\mathrm{CaGa}_{2} \mathrm{~S}_{4}: \mathrm{Tm}^{3+}$ excitation spectrum, an 

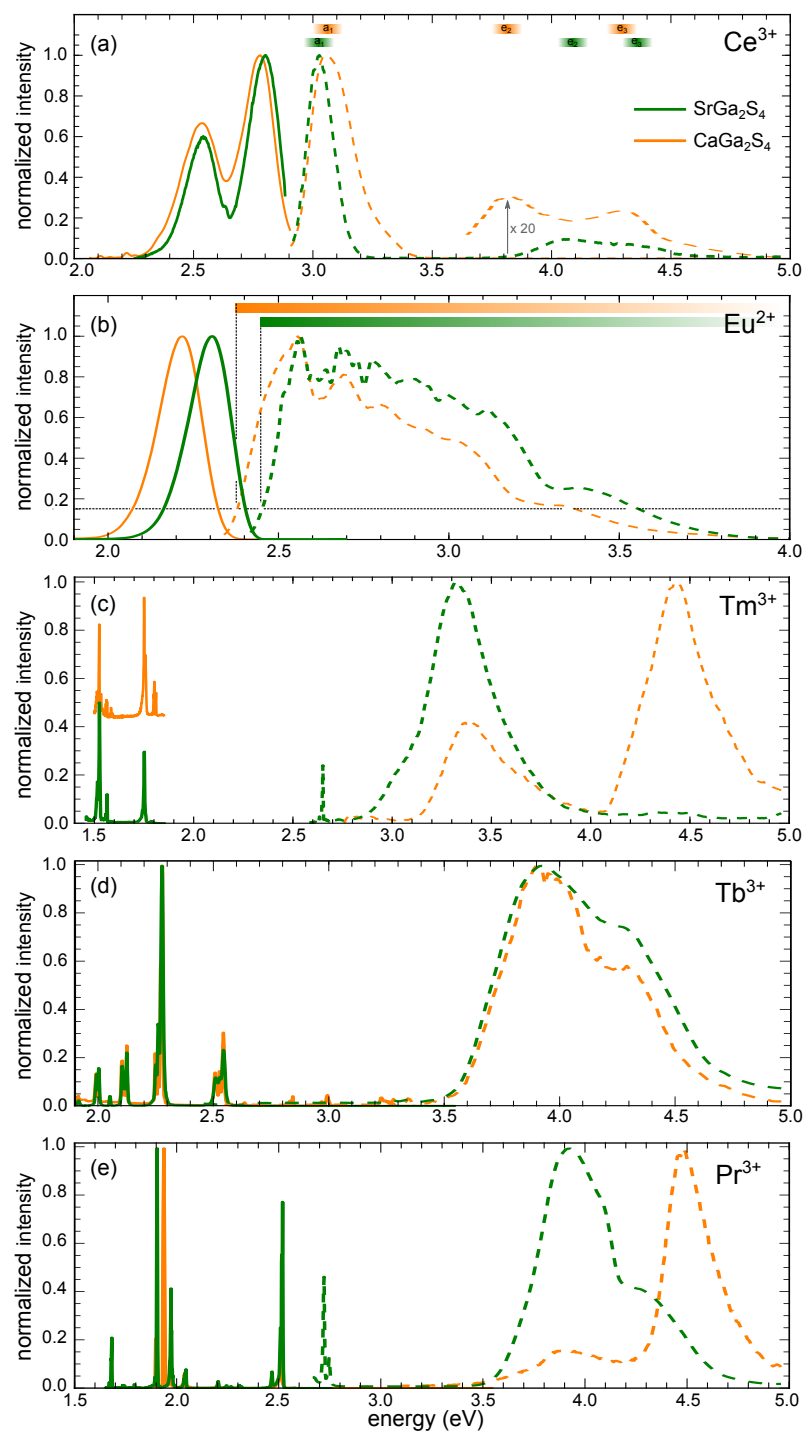

Figure 6 - Emission (solid lines) and excitation spectra (dashed lines) of lanthanide doped $\mathrm{SrGa}_{2} \mathrm{~S}_{4}$ (green lines) and $\mathrm{CaGa}_{2} \mathrm{~S}_{4}$ (orange lines), measured at $75 \mathrm{~K}$. (a) $\mathrm{Ce}^{3+}$ doped, emission spectrum upon $410 \mathrm{~nm}$ (3.02 $\mathrm{eV})$ excitation, excitation spectrum for $445 \mathrm{~nm}(2.79$ eV) emission. (b) $\mathrm{Eu}^{2+}$ doped, emission spectrum upon $450 \mathrm{~nm}(2.76 \mathrm{eV})$ excitation, excitation spectrum for $535 \mathrm{~nm}(2.32 \mathrm{eV})(560 \mathrm{~nm}(2.21 \mathrm{eV}))$ emission for $\mathrm{SrGa}_{2} \mathrm{~S}_{4}\left(\mathrm{CaGa}_{2} \mathrm{~S}_{4}\right)$. (c) $\mathrm{Tm}^{3+}$ doped, emission spectrum upon $370 \mathrm{~nm}(3.35 \mathrm{eV})$ excitation, excitation spectrum for $810 \mathrm{~nm}(1.53 \mathrm{eV})$ emission. (d) $\mathrm{Tb}^{3+}$ doped, emission spectrum upon $302 \mathrm{~nm}(4.11 \mathrm{eV})(320$ $\mathrm{nm}(3.88 \mathrm{eV}))$ excitation, excitation spectrum for 544 $\mathrm{nm}(2.28 \mathrm{eV})$ emission for $\mathrm{SrGa}_{2} \mathrm{~S}_{4}\left(\mathrm{CaGa}_{2} \mathrm{~S}_{4}\right)$. (e) $\mathrm{Pr}^{3+}$ doped, emission spectrum upon $320 \mathrm{~nm}(3.87 \mathrm{eV})$ excitation, excitation spectrum for $651 \mathrm{~nm}(1.90 \mathrm{eV})$ $(640 \mathrm{~nm}(1.94 \mathrm{eV}))$ emission for $\mathrm{SrGa}_{2} \mathrm{~S}_{4}\left(\mathrm{CaGa}_{2} \mathrm{~S}_{4}\right)$. Spectra of $\mathrm{CaGa}_{2} \mathrm{~S}_{4}: \mathrm{Ln}^{Q+}\left(\mathrm{Ln}^{Q+}=\mathrm{Ce}^{3+}, \mathrm{Eu}^{2+}\right.$, $\mathrm{Tm}^{3+}$ ) adapted from [8], reproduced with permission. additional band peaking at $4.43 \mathrm{eV}$ is visible. Upon excitation in this band, a broadband emission appears, peaking around $630 \mathrm{~nm}$ which dominates the $\mathrm{Tm}^{3+}$ emission. A similar excitation band was also reported elsewhere and ascribed to fundamental absorption in the host material, sensitizing the $\mathrm{Tm}^{3+}$ emission [64]. Comparison of the $\mathrm{Tm}^{3+}$ excitation spectrum of a sample prepared in forming gas atmosphere $\left(\mathrm{N}_{2} / \mathrm{H}_{2}\right)$, with a similar sample, though prepared in hydrogen sulfide $\left(\mathrm{H}_{2} \mathrm{~S}\right)$ atmosphere, shows that the relative intensity of the Tm-S charge transfer peak increases in the latter case. This suggests that the additional band can as well originate from sulfur defects, unavoidably present in the prepared materials. A similar excitation band, however less intense, is also visible in the spectrum of $\mathrm{SrGa}_{2} \mathrm{~S}_{4}: \mathrm{Tm}^{3+}$. No further investigation was performed on this spectral feature as it is not directly related to $\mathrm{Tm}^{3+}$ centers or other lanthanide defects.

If the excitation spectra of $\mathrm{Ce}^{3+}$ and $\mathrm{Eu}^{2+}$ are compared for both host materials, a deviation from the idealized situation, described by a linear relationship between the absorption energies of both ions, occurs. The $\mathrm{Eu}^{2+}$ band is redshifted for $\mathrm{CaGa}_{2} \mathrm{~S}_{4}$ with respect to $\mathrm{SrGa}_{2} \mathrm{~S}_{4}$, in agreement with the observed yellow and green emission respectively. This is understood to be due to the increased crystal field strength, i.e. the accumulated effect of the centroid shift and crystal field splitting, due to the decreased bond length in the case of the Ca-compound. In the case of $\mathrm{Ce}^{3+}$, however, both excitation spectra approximately coincide at the low energy side (Fig. 6).

Although this behavior lies fully within the expected uncertainties, it is still remarkable if an isomorphous coordination polyhedron would be expected. However, from the EPR study of Warren et al. (see \$3.2.1) 24], it is known that the situation is locally completely different for $\mathrm{SrGa}_{2} \mathrm{~S}_{4}: \mathrm{Ce}^{3+}$ which can explain this anti-correlation between the calcium and strontium thiogallates.

Typical green $\mathrm{Tb}^{3+}{ }^{5} \mathrm{D}_{4}$ emission is often excited by $4 \mathrm{f}^{8}-4 \mathrm{f}^{7} 5 \mathrm{~d}^{1}$ absorption in the UV range. Remarkably, the excitation spectra of $\mathrm{Tb}^{3+}$, displayed in Fig. 6(d), coincide for doping in $\mathrm{CaGa}_{2} \mathrm{~S}_{4}$ and $\mathrm{SrGa}_{2} \mathrm{~S}_{4}$, which indicates that $\mathrm{Tb}_{\mathrm{Sr}}^{\bullet}$ is likely to be charge-compensated in the same way as $\mathrm{Ce}_{\mathrm{Sr}}^{\bullet}$. Assuming an equal spectroscopic redshift for both lanthanides, the $\mathrm{SrGa}_{2} \mathrm{~S}_{4}: \mathrm{Tb}^{3+}$ spin-allowed and spin-forbidden absorption bands are predicted at respectively $4.68 \mathrm{eV}$ and $3.98 \mathrm{eV}$ 65]. The experimental excitation spectrum (Fig. 6) shows this feature around $3.91 \mathrm{eV}$, most likely corresponding with the spin-forbidden transition. The spin-allowed transition is therefore believed to be obscured by fundamental absorption by the host crystals.

The $\mathrm{Pr}^{3+}-\mathrm{S}^{2-}$ charge transfer is expected at 4.47 $\mathrm{eV} \pm 0.20 \mathrm{eV}$ and the $4 \mathrm{f}^{2}-4 \mathrm{f}^{1} 5 \mathrm{~d}^{1}$ absorption band is expected at $4.54 \pm 0.12 \mathrm{eV}$ in $\mathrm{SrGa}_{2} \mathrm{~S}_{4}$ assuming 
Table 2 - Optical properties of $\mathrm{Ce}^{3+}, \mathrm{Eu}^{2+}$ and $\mathrm{Tm}^{3+}$ ions in the $\mathrm{MGa}_{2} \mathrm{~S}_{4}$ hosts. These parameters serve as input for the calculation of the lanthanide energy level schemes. $d_{1}, d_{2}$ and $d_{3}$ denote the positions of the three 4f- $5 \mathrm{~d}$ excitation bands of $\mathrm{Ce}^{3+}, \mathrm{f}_{1}$ and $\mathrm{f}_{2}$ denote the positions of the two $5 \mathrm{~d}-4 \mathrm{f}$ emission bands of $\mathrm{Ce}^{3+}$. Data for $\mathrm{CaGa}_{2} \mathrm{~S}_{4}: \mathrm{Ln}^{Q+}$ adapted from 8, reproduced with permission. The motivation for the experimental uncertainties is explained herein. All values in $\mathrm{eV}$.

\begin{tabular}{|c|c|c|c|}
\hline & & $A=\mathrm{SrGa}_{2} \mathrm{~S}_{4}$ & $A=\mathrm{CaGa}_{2} \mathrm{~S}_{4}$ \\
\hline & Optical band gap $E^{e x}(A)$ & $4.16 \pm 0.10$ & $3.98 \pm 0.10$ \\
\hline \multirow[t]{9}{*}{$\mathbf{C e}^{3+}$} & Absorption $E_{\mathrm{abs}}^{\mathrm{fd}_{1}}\left(\mathrm{Ce}^{3+}, A\right)$ & $3.03 \pm 0.02$ & $3.05 \pm 0.02$ \\
\hline & Absorption $E_{\mathrm{abs}}^{\mathrm{td}}\left(\mathrm{Ce}^{3+}, A\right)$ & $4.04 \pm 0.02$ & $3.81 \pm 0.02$ \\
\hline & Absorption $E_{\mathrm{abs}}^{\mathrm{td}}\left(\mathrm{Ce}^{3+}, A\right)$ & $4.32 \pm 0.02$ & $4.29 \pm 0.02$ \\
\hline & Redshift $D\left(\mathrm{Ce}^{3+}, A\right)$ & $3.09 \pm 0.03$ & $3.07 \pm 0.03$ \\
\hline & Centroid shift $\epsilon_{\mathrm{C}}\left(\mathrm{Ce}^{3+}, A\right)$ & $2.40 \pm 0.02$ & $2.50 \pm 0.02$ \\
\hline & Crystal field splitting $\epsilon_{\mathrm{cfs}}\left(\mathrm{Ce}^{3+}, A\right)$ & $1.29 \pm 0.03$ & $1.24 \pm 0.03$ \\
\hline & Emission $E_{\mathrm{em}}^{\mathrm{f}_{1} \mathrm{~d}}\left(\mathrm{Ce}^{3+}, A\right)$ & $2.54 \pm 0.02$ & $2.54 \pm 0.02$ \\
\hline & Emission $E_{\mathrm{em}}^{\mathrm{f}_{2} \mathrm{~d}}\left(\mathrm{Ce}^{3+}, A\right)$ & $2.80 \pm 0.02$ & $2.78 \pm 0.02$ \\
\hline & Stokes shift $\Delta S\left(\mathrm{Ce}^{3+}, A\right)$ & $0.23 \pm 0.03$ & $0.27 \pm 0.03$ \\
\hline \multirow[t]{2}{*}{$\mathbf{E u}^{2+}$} & Redshift $D\left(\mathrm{Eu}^{2+}, A\right)$ & $1.76 \pm 0.05$ & $1.83 \pm 0.05$ \\
\hline & Stokes shift $\Delta S\left(\mathrm{Eu}^{2+}, A\right)$ & $0.16 \pm 0.06$ & $0.17 \pm 0.06$ \\
\hline $\mathbf{T m}^{3+}$ & Charge transfer $E^{\mathrm{CT}}\left(\mathrm{Tm}^{3+}, A\right)$ & $3.32 \pm 0.02$ & $3.37 \pm 0.02$ \\
\hline
\end{tabular}

isostructural $\operatorname{Pr}_{\mathrm{Sr}}^{\bullet}$ and $\mathrm{Ce}_{\mathrm{Sr}}^{\bullet}$ defects. Experimentally, broadband excitation peaks are found at lower energy, peaking around at $3.92 \mathrm{eV}$ and $4.32 \mathrm{eV}$ (Fig. 6(e)). If the lowest energy band is assigned to the chargetransfer band and the higher energy band to the $4 \mathrm{f}^{2}-4 \mathrm{f}^{1} 5 \mathrm{~d}^{1}$ transition, the mismatch between prediction and experiment is respectively $2.7 \sigma_{\mathrm{CT}}$ and $1.8 \sigma_{\mathrm{fd}}$ 8. These are rather large deviations, suggesting that possibly a different mechanism causes the origin of the excitation bands. Yet another distorted coordination polyhedron is a possible alternative explanation.

In 2004, Iwamaru et al. studied the cathodoluminescence of $\mathrm{SrGa}_{2} \mathrm{~S}_{4}: \mathrm{Sm}$ 66. They found a broad red emission band about $700 \mathrm{~nm}(1.77 \mathrm{eV})$ which was attributed to $4 \mathrm{f}^{5} 5 \mathrm{~d}^{1}-4 \mathrm{f}^{6}$ emission of $\mathrm{Sm}^{2+}$. From the $\mathrm{Eu}^{2+}$ spectra, the absorption threshold of $\mathrm{Sm}^{2+}$ in $\mathrm{SrGa}_{2} \mathrm{~S}_{4}$ is estimated at $1.25 \mathrm{eV}$ and the emission at $1.09 \mathrm{eV}$. Therefore, it is unlikely that the observed emission band is due to $\mathrm{Sm}^{2+}$. From the approximate location of the $2+/ 3+$ charge-state transition level of $\mathrm{Sm}$ (see Fig. 7), it is moreover expected that $\mathrm{Sm}$ will be preferentially incorporated in a trivalent state, assuming that the electronic chemical potential lies in the middle of the band gap 67, 8. This was also clear from the $\mathrm{SrGa}_{2} \mathrm{~S}_{4}: \mathrm{Sm}^{3+} \mathrm{PL}$ spectrum which consists exclusively of characteristic $\mathrm{Sm}^{3+}$ features (not shown).

\subsubsection{Energy level schemes}

Because the deviating Ce incorporation, the chargestate transition level scheme for $\mathrm{SrGa}_{2} \mathrm{~S}_{4}$ cannot be simply constructed based on $E^{e x}(A), E_{\text {abs }}^{\mathrm{fd}_{1}}\left(\mathrm{Ce}^{3+}, A\right)$, $E_{\mathrm{abs}}^{\mathrm{fd}}\left(\mathrm{Eu}^{2+}, A\right)$ and $E^{\mathrm{CT}}\left(\mathrm{Tm}^{3+}, A\right)$, as was done in the case of $\mathrm{CaGa}_{2} \mathrm{~S}_{4}[8]$.

The Coulomb 4f correlation parameter for $\mathrm{Eu}$, $U(\mathrm{Eu}, A)$ which is a key parameter for constructing vacuum referred binding energy level schemes, is as- sessed from the $\mathrm{Ce}^{3+}$ centroid shift, $\epsilon_{\mathrm{c}}\left(\mathrm{Ce}^{3+}, A\right)$ :

$$
U(\mathrm{Eu}, A)=5.44+2.834 \mathrm{e}^{-\epsilon_{\mathrm{c}}\left(\mathrm{Ce}^{3+}, A\right) / 2.2}
$$

This relationship cannot be used in this case because of the different defect geometries for Ce and Eu. Since this and other equations are completely empirical, it is unknown to which extent different charge compensating effects or other imperfections are present in the data used to obtain these equations. It is more specifically possible that this kind of effects is readily accounted for in the empirical routine without the users knowledge. Inspection of the data used for deriving Eq. 1 learns for instance that $\mathrm{SrGa}_{2} \mathrm{~S}_{4}$ was used as a data point 26 .

Concerning this question, it can also be of interest to take a look at the uncertainties, associated with the empirical rules. Eq. 1 allows to calculate $U(\mathrm{Eu}, A)$ with a $68 \%$ uncertainty interval of $100 \mathrm{meV}$. This is of the same order of magnitude as the deviations between $U(\mathrm{Eu}, A)$ between two different defect sites in the same host, based on the above examples. As the occurrence of multiple nonequivalent defects nor discontinuous behavior such as different chargecompensation schemes were systematically taken into account in devising the set of empirical rules, the uncertainties on these empirical rules, as calculated in 8, are larger than differences between possible nonequivalent defects.

Of course, applying the empirical rules in a thoughtful way by making the distinction between different defect geometries will yield more information about the relative positions of the charge-state transition levels for each nonequivalent defect, allowing a comparison between the different physical observables associated with them.

Assuming that the $\mathrm{Ce}_{\mathrm{Sr}}^{\bullet}$ and $\mathrm{Tm}_{\mathrm{Sr}}^{\bullet}$ defects are isostructural in $\mathrm{SrGa}_{2} \mathrm{~S}_{4}$, host referred and vacuum referred binding energy level schemes for the defect 


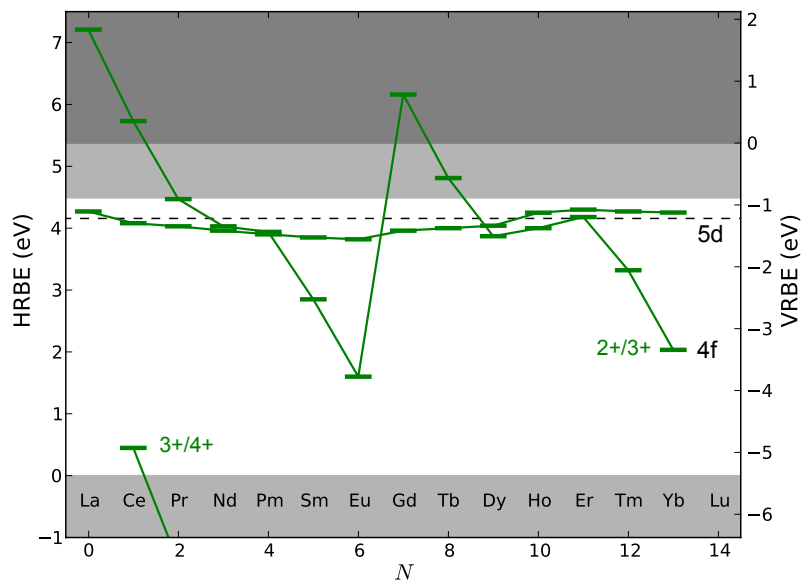

Figure 7 - Diagram with the $2+/ 3+$ and $3+/ 4+$ charge-state transition levels for the $\mathrm{Ln}_{\mathrm{Sr}}$ defects which are isostructural to the $\mathrm{Ce}_{\mathrm{Sr}}^{\bullet}$ defect in $\mathrm{SrGa}_{2} \mathrm{~S}_{4}$.

with nearby sulfur interstitial can be constructed according to the usual routine. The result is displayed in Fig. 7.

Obtaining a charge-state transition level scheme for the 8-fold coordinated lanthanide defects in $\mathrm{SrGa}_{2} \mathrm{~S}_{4}$ such as the $\mathrm{Eu}_{\mathrm{Sr}}$ defect is not straightforward as neither the $\mathrm{Ce}^{3+}$ centroid shift can be used to obtain vacuum referred binding energies nor a charge-transfer energy of a trivalent lanthanide to probe host referred binding energies. Both rely on spectroscopy of trivalent lanthanides which form different defect geometries. The only remaining possibility is to address core-hole spectroscopic techniques such as photo-electron spectroscopy.

\section{Conclusions}

In this manuscript, possible refinements of the set of empirical rules used to construct charge-state transition level schemes for lanthanide materials have been discussed. More specifically, it was investigated to what extent the occurrence of multiple nonequivalent defects within the lanthanide series alters the level schemes.

Following reports in literature containing energy level schemes, it is commonly assumed that only one nonequivalent defect is present in a certain host which is isostructural for each lanthanide dopant, whatever its formal charge. The validity of this assumption is doubtful, however a painstaking task to confirm experimentally as it involves doping with a large number of lanthanide ions and possibly specialized analytical techniques such as electron paramagnetic resonance or X-ray absorption spectroscopy. In this article, a few cases were discussed where the above assumption is known to be invalid. A distinction between two situations was made.

First, host compounds were considered in which multiple nonequivalent substitutional defects occur. $\mathrm{SrAl}_{2} \mathrm{O}_{4}$ and $\mathrm{Sr}_{2} \mathrm{Si}_{5} \mathrm{~N}_{8}$ are examples for which the lanthanide ions spontaneously occupy both $\mathrm{Sr}$ sites. In these cases, clear spectroscopic differences are visible and site-selective spectroscopy allows to construct independent charge-state transition level schemes for each defect geometry. From these examples, it is clear that very limited structural differences of coordination environment can give rise to important deviations in the optical spectra.

Second, host compounds were considered for which different lanthanide ions show a different behavior with respect to the local coordination. $\mathrm{SrGa}_{2} \mathrm{~S}_{4}$ is an example in which $\mathrm{Ce}^{3+}$ and $\mathrm{Eu}^{2+}$ form very different defect geometries. If this is the case a discontinuity across the lanthanide series exists and the empirical rules exploiting the systematics between the different ions ions cannot be used to construct charge-state transition level schemes. Therefore, only partial schemes can be made.

The energy differences between the charge-state transition levels and energy levels belonging to the same lanthanide in a different defect geometry were compared with the typical uncertainties of the empirical models. It is concluded that both are of the same order of magnitude. This could imply that some of the data used to derive the empirical rules are compromised, if multiple nonequivalent defects occur or if structural distortions across the lanthanide series were not identified. Consequently, the error margins related to the present empirical rules might potentially be reduced if the underlying input data is scrutinized.

\section{Acknowledgements}

JJJ thanks the agency for Innovation by Science and Technology (IWT) for a research grant (121024). PFS and DP are grateful to the IWT for a SBO-IWT grant (SBO130030). Olivier Janssens is acknowledged for the assistance with the XRD measurements.

\section{References}

\section{References}

[1] M. Gaft, R. Reisfeld, and G. Panczer. Modern Luminescence Spectroscopy of Minerals and $\mathrm{Ma}$ terials. Springer, 2005.

[2] M. H. Crawford. IEEE Journal of Selected Topics in Quantum Electronics, 15(4):1028-1040, 2009.

[3] Rong-Jun Xie, N. Hirosaki, and T. Takeda. Applied Physics Express, 2(2):022401, 2009.

[4] S. Ye, F. Xiao, Y. X. Pan, Y. Y. Ma, and Q. Y. Zhang. Materials Science \& Engineering R-Reports, 71(1):1-34, 2010. 
[5] P. F. Smet, A. B. Parmentier, and D. Poelman. Journal of the Electrochemical Society, 158(6):R37-R54, 2011.

[6] A. Jha, B. Richards, G. Jose, T. Teddy-Fernandez, P. Joshi, X. Jiang, and J. Lousteau. Progress in Materials Science, 57(8):1426-1491, 2012.

[7] R.J.D. Tilley. Defects in Solids. Wiley, 2008.

[8] J. J. Joos, D. Poelman, and P. F. Smet. Physical Chemistry Chemical Physics, 17(29):19058-19078, 2015.

[9] M. Dolg. Computational Methods in Lanthanide and Actinide Chemistry. Wiley, 2015.

[10] K.A. Kikoin and V.N. Fleurov. Transition Metal Impurities in Semiconductors, Electronic Structure and Physical Properties. World Scientific, 1994.

[11] P. Dorenbos. ECS Journal of Solid State Science and Technology, 2(2):R3001-R3011, 2013.

[12] C. Freysoldt, B. Grabowski, T. Hickel, J. Neugebauer, G. Kresse, A. Janotti, and C. G. Van de Walle. Reviews of Modern Physics, 86(1):253-305, 2014.

[13] C. W. Thiel, H. Cruguel, Y. Sun, G. J. Lapeyre, R. M. Macfarlane, R. W. Equall, and R. L. Cone. Journal of Luminescence, 94:1-6, 2001.

[14] C. W. Thiel. Thesis, 2003.

[15] P. Dorenbos. Journal of Materials Chemistry, 22(42):22344-22349, 2012.

[16] Z. J. Zhang, A. Feng, X. Y. Chen, and J. T. Zhao. Journal of Applied Physics, 114(21):213518, 2013.

[17] A. B. Parmentier, J. J. Joos, P. F. Smet, and D. Poelman. Journal of Luminescence, 154:445451, 2014.

[18] VítJarý, Lubomír Havlák, Jan Bárta, Maksym Buryi, Eva Mihóková, Martin Rejman, Valentin Laguta, and Martin Nikl. Materials, 8(10):5348, 2015 .

[19] J. Ueda, P. Dorenbos, A. J. J. Bos, K. Kuroishi, and S. Tanabe. Journal of Materials Chemistry C, 3(22):5642-5651, 2015.

[20] L. X. Ning, C. C. Zhou, W. P. Chen, Y. C. Huang, C. K. Duan, P. Dorenbos, Y. Tao, and H. B. Liang. Journal of Physical Chemistry C, 119(12):67856792, 2015.

[21] A. D. Sontakke, J. Ueda, Y. Katayama, P. Dorenbos, and S. Tanabe. Applied Physics Letters, 106(13):131906, 2015.

[22] J. He, R. Shi, M. G. Brik, P. Dorenbos, Y. Huang, Y. Tao, and H. B. Liang. Journal of Luminescence, 161:257-263, 2015.
[23] A. Dobrowolska, E. C. Karsu, A. J. J. Bos, and P. Dorenbos. Journal of Luminescence, 160:321$327,2015$.

[24] W. L. Warren, K. Vanheusden, M. A. Rodriguez, C. H. Seager, D. R. Tallant, P. D. Rack, P. H. Holloway, B. K. Wagner, C. J. Summers, and P. N. Yocom. Applied Physics Letters, 70(4):478-480, 1997.

[25] P. Dorenbos. Physical Review B, 85(16):165107, 2012.

[26] P. Dorenbos. Journal of Luminescence, 135(0):93104, 2013.

[27] P. Dorenbos. Journal of Physics-Condensed Matter, 15(49):8417-8434, 2003.

[28] T. Matsuzawa, Y. Aoki, N. Takeuchi, and Y. Murayama. Journal of the Electrochemical Society, 143(8):2670-2673, 1996.

[29] K. Van den Eeckhout, P. F. Smet, and D. Poelman. Materials, 3(4):2536-2566, 2010.

[30] K. Korthout, K. Van den Eeckhout, J. Botterman, S. Nikitenko, D. Poelman, and P. F. Smet. Physical Review B, 84(8):085140, 2011.

[31] J. Botterman, J. J. Joos, and P. F. Smet. Physical Review B, 90(8):085147, 2014.

[32] S. H. M. Poort, W. P. Blokpoel, and G. Blasse. Chemistry of Materials, 7(8):1547-1551, 1995.

[33] T. Aitasalo, J. Holsa, H. Jungner, J. C. Krupa, M. Lastusaari, J. Legendziewicz, and J. Niitykoski. Radiation Measurements, 38(4-6):727730,2004 .

[34] F. Clabau, X. Rocquefelte, S. Jobic, P. Deniard, M. H. Whangbo, A. Garcia, and T. Le Mercier. Chemistry of Materials, 17(15):3904-3912, 2005.

[35] J. Hölsä, T. Laamanen, M. Lastusaari, M. Malkamki, and $\mathrm{P}$ Novk. Desy annual report, 2009.

[36] D. D. Jia, X. J. Wang, W. Jia, and W. M. Yen. Journal of Luminescence, 119:55-58, 2006.

[37] Y. Q. Li, G. de With, and H. T. Hintzen. Journal of Luminescence, 116(1-2):107-116, 2006.

[38] Agata Lazarowska, Sebastian Mahlik, Marek Grinberg, Chiao-Wen Yeh, and Ru-Shi Liu. Optical Materials, 37(0):734-739, 2014.

[39] Y. Q. Li, J. E. J. van Steen, J. W. H. van Krevel, G. Botty, A. C. A. Delsing, F. J. DiSalvo, G. de With, and H. T. Hintzen. Journal of Alloys and Compounds, 417(1-2):273-279, 2006.

[40] X. Q. Piao, T. Horikawa, H. Hanzawa, and K. Machida. Applied Physics Letters, 88(16):161908, 2006. 
[41] R. J. Xie, N. Hirosaki, N. Kimura, K. Sakuma, and M. Mitomo. Applied Physics Letters, 90(19):191101, 2007.

[42] S. E. Brinkley, N. Pfaff, K. A. Denault, Z. J. Zhang, H. T. Hintzen, R. Seshadri, S. Nakamura, and S. P. DenBaars. Applied Physics Letters, 99(24):241106, 2011.

[43] R.-J. Xie and H. T. Hintzen. Journal of the American Ceramic Society, 96(3):665-687, 2013.

[44] Rong-Jun Xie, Naoto Hirosaki, T. Takeda, and T. Suehiro. ECS Journal of Solid State Science and Technology, 2(2):R3031-R3040, 2013.

[45] R.-J. Xie and H. T. Hintzen. Journal of the American Ceramic Society, 96(3):665-687, 2013.

[46] O. M. ten Kate, Z. Zhang, P. Dorenbos, H. T. Hintzen, and E. van der Kolk. Journal of Solid State Chemistry, 197:209-217, 2013.

[47] A. Zych, J. Ogieglo, C. Ronda, C. D. Donega, and A. Meijerink. Journal of Luminescence, 134:174179, 2013.

[48] T. Schlieper, W. Milius, and W. Schnick. Zeitschrift Fur Anorganische Und Allgemeine Chemie, 621(8):1380-1384, 1995.

[49] A. R. Schulze and H. Müller-Buschbaum. Zeitschrift Fur Anorganische Und Allgemeine Chemie, 475(4):205-210, 1981.

[50] R. Hoppe. Zeitschrift Fur Kristallographie, 150(14):23-52, 1979. Jf409 Times Cited:512 Cited References Count:50.

[51] R. Shi, M. M. Qi, L. X. Ning, F. J. Pan, L. Zhou, W. J. Zhou, Y. C. Huang, and H. B. Liang. Journal of Physical Chemistry C, 119(33):1932619332, 2015.

[52] C. W. Yeh, W. T. Chen, R. S. Liu, S. F. Hu, H. S. Sheu, J. M. Chen, and H. T. Hintzen. Journal of the American Chemical Society, 134(34):1410814117, 2012.

[53] R. D. Shannon. Acta Crystallographica Section A, 32(Sep1):751-767, 1976.

[54] R. Roques, R. Rimet, J. P. Declercq, and G. Germain. Acta Crystallographica Section B-Structural Science, 35(Mar):555-557, 1979.

[55] Jonas J. Joos, Katleen Korthout, Sergey Nikitenko, Dirk Poelman, and Philippe F. Smet. Opt. Mater. Express, 3(9):1338-1350, 2013.

[56] P. Benalloul, C. Barthou, and J. Benoit. Journal of Alloys and Compounds, 275:709-715, 1998.

[57] J. C. Souriau, Y. D. Jiang, J. Penczek, H. G. Paris, and C. J. Summers. Materials Science and Engineering B-Solid State Materials for Advanced Technology, 76(2):165-168, 2000.
[58] C. Chartier, P. Benalloul, C. Barthou, J. M. Frigerio, G. O. Mueller, R. Mueller-Mach, and T. Trottier. Journal of Physics D-Applied Physics, 35(4):363-368, 2002.

[59] C. Chartier, C. Barthou, P. Benalloul, and J. M. Frigerio. Journal of Luminescence, 111(3):147$158,2005$.

[60] Y. Arai, H. Kominami, Y. Nakanishi, and Y. Hatanaka. Applied Surface Science, 244(14):473-476, 2005.

[61] J. J. Joos, K. W. Meert, A. B. Parmentier, D. Poelman, and P. F. Smet. Optical Materials, 34(11):1902-1907, 2012.

[62] B. Eisenmann, M. Jakowski, W. Klee, and H. Schafer. Revue De Chimie Minerale, 20(2):255263, 1983.

[63] I. Kitajima, T. Takizawa, C. Hidaka, and S. Nomura. Physica Status Solidi C: Current Topics in Solid State Physics, Vol 10, No 7-8, 10(7-8):11191122, 2013.

[64] A. Bessière, P. Dorenbos, C. W. E. van Eijk, E. Yamagishi, C. Hidaka, and T. Takizawa. Journal of the Electrochemical Society, 151(12):H254H260, 2004.

[65] P. Dorenbos. Journal of Physics-Condensed Matter, 15(36):6249-6268, 2003.

[66] M. Iwamaru, H. Nakajima, H. Kominami, Y. Nakanishi, and Y. Hatanaka. Journal of Vacuum Science \& Technology B, 22(3):1382-1385, 2004.

[67] P. Dorenbos. Chemistry of Materials, 17(25):6452-6456, 2005. 